\title{
Flow Cytometric Analysis of ZAP-70 Protein Expression for B-Cell Chronic Lymphocytic Leukemia Prognostication: Usefulness and Limitations
}

\author{
Muhammad Shariq Shaikh ${ }^{1}$, Arsalan Ahmed ${ }^{1}$, Summaiya Sohail ${ }^{1}$, Amin Fahim ${ }^{2}$, Sadiq Hussain Nohario ${ }^{3}$ \\ , Shahid Pervez ${ }^{1}$ \\ 1. Pathology and Laboratory Medicine, Aga Khan University Hospital, Karachi, PAK 2. Pathology, Isra University, \\ Hyderabad, PAK 3. Oncology, Nuclear Institute of Medicine and Radiotherapy, Jamshoro, PAK
}

Corresponding author: Muhammad Shariq Shaikh, muhammad.shariq@aku.edu

\begin{abstract}
Objectives: The heterogenous clinical course in B-cell chronic lymphocytic leukemia (B-CLL) can be linked to several genetic and phenotypic characteristics of malignant B-cells. Prognostic analysis in B-CLL is routinely carried out to assist patient management; particularly to predict the time to initiate treatment. Increased ZAP-70 expression is a surrogate marker for unmutated immunoglobulin genes and inferior clinical outcomes which can be quantified to predict future outcomes in B-CLL patients. The study determined the ZAP-70 expression pattern using Z-index in Pakistani patients with B-CLL.
\end{abstract}

Methods: A retrospective analysis of B-CLL cases diagnosed and confirmed on flow cytometry at Aga Khan University Hospital for the last six years which had also undergone ZAP-70 analysis were included. In all these cases, ZAP-70 expression was quantified by measuring mean fluorescence intensities (MFIs) of normal B-cells, T-cells, and CLL-cells (CD19 and CD5 double-positive population). ZAP-70 expression was divided into high, low, and negative categories based on Z-index calculation. Mann-Whitney U test was utilized to determine the significance of ZAP-70 variations in different age groups and genders. P-value $<0.05$ was considered significant.

Results: A total of 120 patients of B-CLL had ZAP-70 analysis during the study period. The median age was 62 with an interquartile range of 35-87 and male to female ratio of 2:1. ZAP-70 expression was high in 18 (15\%), low in 52 (43.3\%) and negative in 50 (41.7\%) cases. No significant difference in ZAP-70 expression with respect to the age or gender of the study population was identified using appropriate statistical calculations.

Review began 10/24/2020 Review ended 11/14/2020 Published 11/24/2020

\section{(c) Copyright 2020}

Shaikh et al. This is an open access article distributed under the terms of the Creative Commons Attribution License CC-BY 4.0., which permits unrestricted use, distribution, and reproduction in any medium, provided the original author and source are credited.
Conclusions: This study showed only 15\% of B-CLL cases showing high ZAP-70 expression, a surrogate biomarker for possible aggressive behavior which may necessitate therapeutic intervention and close surveillance.

Categories: Pathology, Oncology, Hematology

Keywords: b-cell chronic lymphocytic leukemia (b-cll), zap-70, flow cytometry, pakistan

\section{Introduction}

Chronic lymphocytic leukemia/small lymphocytic lymphoma (CLL/SLL) is a neoplasm characterized by progressive accumulation of small monomorphic mature B-lymphocytes that are functionally ineffective. In the absence of extramedullary tissue involvement (small lymphocytic lymphoma (SLL)), there must be $\geqslant 5 \mathrm{x}$ 109/L monoclonal B-lymphocytes with a CLL phenotype. Accounting for around 25\% of all leukemias in the United States, it is the most common type of leukemia in Western adults [1].

The heterogeneity of CLL in terms of disease stage at diagnosis, cytogenetic abnormalities, biochemical and other markers is reflected in substantially different clinical outcomes among cases. Survival widely varies after diagnosis anywhere from 2-20 years with a median of 10 years [2,3]. This poses a great dilemma for treating physicians if and when to intervene. Prognostic bio-markers include lymphocyte doubling time, beta-2 microglobulin levels, expression of CD38, zeta chain associated (ZAP-70) proteins, immunoglobulin heavy-chain gene (IgHV) mutation status, and various other genetic abnormalities [4-6].

ZAP-70 is a tyrosine kinase normally required for T-cell differentiation and function. Normally, absent in Blymphocytes, the increased expression in B-CLL is reported to be associated with poor prognosis [7]. Although discordance rates of up to $20 \%$ have been reported in various studies, the increased level of ZAP70 is considered to be the most important discriminating gene between unmutated and mutated IgHV types of CLL. ZAP-70 possibly acts by decreasing the threshold for signaling through bcl2, hence facilitating its 
antiapoptotic effects [8].

ZAP-70 expression may be assessed by various methods including immunohistochemistry, however multiparameter flow cytometry (MFC) is a sophisticated technique that can readily be used to study various cellular characteristics simultaneously and objectively hence ZAP-70 expression analysis by MFC is a very useful rapid tool to determine prognosis in CLL. Mean fluorescence intensity (MFI) of anti-ZAP-70 labeled fluorochrome is calculated in CLL cells (CD19+, CD5+), normal B-cells (CD19+, CD5-), and T-cells (CD19-, $\mathrm{CD} 5+)$. Relative intensities of expression in different cell subtypes can be used to establish positive and negative thresholds. Most studies done in the past have described ZAP-70 expression as positive or negative using distinct cut-offs [9]. A significant proportion of patients exist where ZAP-70 expression is in the gray zone. This cohort of patients must be identified using appropriate methodology and calculations and their clinical outcome correlated. This group of patients might explain the indeterminate prognostic group in whom the initial indolent course of B-CLL may abruptly transform into an aggressive phase and a rapid downhill course. Literature review revealed a lack of data focusing on low (gray zone) ZAP-70 expression in B-CLL. The current study is aimed at determining the pattern of ZAP-70 expression using Z-index in Pakistani B-CLL patients in order to have an insight about the proportion of gray zone (low expression) cases in addition to distinct positive and negative cases.

\section{Materials And Methods}

\section{Study setting}

The clinical laboratories of Aga Khan University Hospital (AKUH) serve as a referral center for the country. The laboratory is ISO 9001:2008 certified, accredited by Joint Commission International Accreditation (JCIA) and College of American Pathologist (CAP). The flow cytometry service caters for both in-patient and outside referrals with significant volumes. This retrospective analysis was performed at the Department of Pathology \& Laboratory Medicine, AKUH. All B-CLL cases diagnosed and confirmed on flow cytometry for six consecutive years (2014-2019) on which ZAP-70 analysis was also done were included.

\section{Confirmation of B-CLL on flow cytometry}

In all cases, the diagnosis of B-CLL was confirmed immunophenotypically by flow cytometry. Gating was done on a bright CD45 positive lymphocyte population. The comprehensive panel of antibodies included: Bcell antigens (CD19, CD20, CD22, CD23, CD79a, FMC7), T-cell antigens (cCD3, CD3, CD5, CD7), myeloid antigens (MPO, CD13, CD33), markers of immaturity (CD34, Tdt), markers of clonality (kappa and lambda), and others (HLA-DR, CD117, CD10). For both B-CLL diagnosis and ZAP-70 expression analysis, FC500 MCL Flow Cytometer (Beckman Coulter, Miami, FL, USA) was used.

\section{ZAP-70 expression analysis}

Whole blood samples were collected in an EDTA tube and processed within $24 \mathrm{~h}$ after collection. Commercially prepared ZAP-70-phycoerythrin antibodies (Beckman Coulter Life Sciences) were used along with CD5-fluorescein isothiocyanate), CD19-phycoerythrin-cyanin 5.1, and CD3+CD56 phycoerythrincyanine 7. MFI of ZAP-70 was measured in normal B-cells (CD19+, CD5-), normal T-cells (CD3+, CD5+), and B-CLL cells (CD19+, CD5+). Z-index was calculated as follows:

Z-index = B-CLL MFI - B-cell MFI X 100 / T-cell MFI - B-cell MFI

As extensively optimized in a landmark study [10], the Z-index value of $\geqslant 27$ was considered as high, $\leqslant 0.3$ as negative, and low if falls between these two values.

\section{Data analysis}

Statistical Package for Social Science version 21 (IBM Corp., Armonk, NY, USA) was used for statistical analysis. Descriptive statistics were computed for all quantitative variables in terms of the median and interquartile range (IQR). The frequency with percentages was computed for qualitative characteristics. Shapiro-Wilk test was applied to determine the normality assumptions for all Z-index values. In case, normality assumption was not met, the Mann-Whitney U test was used. Mann-Whitney U test was also used to determine the significance of Z-index variation between different age groups and genders. The p-value $<0.05$ was taken as significance.

\section{Ethical issues}

An ethical exemption to conduct this analysis was granted by the Ethical Review Committee, Aga Khan University Hospital (\#0404-290). Relevant counseling regarding the prognostic impact of the ZAP-70 results was provided to all who followed up later in the outpatient department.

\section{Results}

A total of 120 B-CLL cases on which ZAP-70 expression was analyzed included 81 males and 39 females with 


\section{Cureus}

an $M: F=2: 1$. The median age of the study population was 62 years with an interquartile range of $35-87$, it was $62(40-87)$ years for males and 61 (35-75) years for females. All the cases had acceptable viability index above the cut-off of $70 \%$ therefore, all the cases were included for the analysis (mean \pm SD viability was $94.43 \pm 5.21 \%)$. ZAP-70 expression was high in 18 (15\%), low in 52 (43.3\%), and negative in 50 (41.7\%) cases. $\mathrm{Z}$-index value in males and females showed no significant difference (Table 1).

\begin{tabular}{|c|c|c|c|c|c|c|c|}
\hline \multirow{2}{*}{ Z-index } & \multicolumn{2}{|c|}{ Males $(n=81)$} & \multicolumn{2}{|c|}{ Females $(n=39)$} & \multicolumn{2}{|l|}{ Total } & \multirow{2}{*}{ P-value } \\
\hline & n (\%) & Median [IQR] & $\mathrm{n}(\%)$ & Median [IQR] & $n(\%)$ & Median [IQR] & \\
\hline High (>27) & $11(13.6)$ & $93.9[62.3-142.9]$ & $7(17.9)$ & 29.7 [27.6-93.0] & $18(15)$ & 79.5 [29.7-112.4] & 0.08 \\
\hline Low $(0.4-27)$ & $37(45.7)$ & $13.3[10.1-21.2]$ & $15(38.4)$ & $12.0[9.2-18.8]$ & $52(43.3)$ & $12.8[9.8-20.8]$ & 0.51 \\
\hline Negative $(\leq 0.3)$ & $33(40.7)$ & $2.7[1.5-3.4]$ & $17(43.7)$ & $2.0[1.2-4.0]$ & $50(41.7)$ & $2.5[1.3-4.0]$ & 0.84 \\
\hline
\end{tabular}

TABLE 1: Median and Inter Quartile Z-Index Values in Males and Females

Sixty-eight (56.7\%) patients were above the age of 60 years. The Z-index analysis showed no significant difference in patients older than 60 years from patients $\leqslant 60$ years of age (Table 2 ).

\begin{tabular}{|l|lll|ll|l|l|}
\hline \multicolumn{2}{|l}{ Age $\leq 60$ years } & & Age $>60$ years & & Total & P-value \\
& $\mathrm{n}(\%)$ & Median [IQR] & $\mathrm{n}(\%)$ & Median [IQR] & $\mathrm{n}(\%)$ & Median [IQR] & \\
High (>27) & $10(19.2)$ & $93.5[67.3-112.4]$ & $8(11.8)$ & $44.9[28.9-102.6]$ & $18(15)$ & $79.5[29.7-112.4]$ & 0.23 \\
Low (0.4-27) & $24(46.2)$ & $11.9[10.3-20.5]$ & $28(41.2)$ & $13.4[9.0-21]$ & $52(43.3)$ & $12.8[9.8-20.8]$ & 0.95 \\
Negative $(\leq 0.3)$ & $18(34.6)$ & $3.4[1.3-4.1]$ & $32(47.1)$ & $2.3[1.3-3.4]$ & $50(41.7)$ & $2.5[1.3-4.0]$ & 0.46 \\
\hline
\end{tabular}

TABLE 2: Median and Inter Quartile Z-Index Values in $\leq 60$ Years and $>60$ Years Patients

Figure 1 shows cases with negative, low, and high ZAP-70 protein expression in B-CLL cases. 


\section{Cureus}

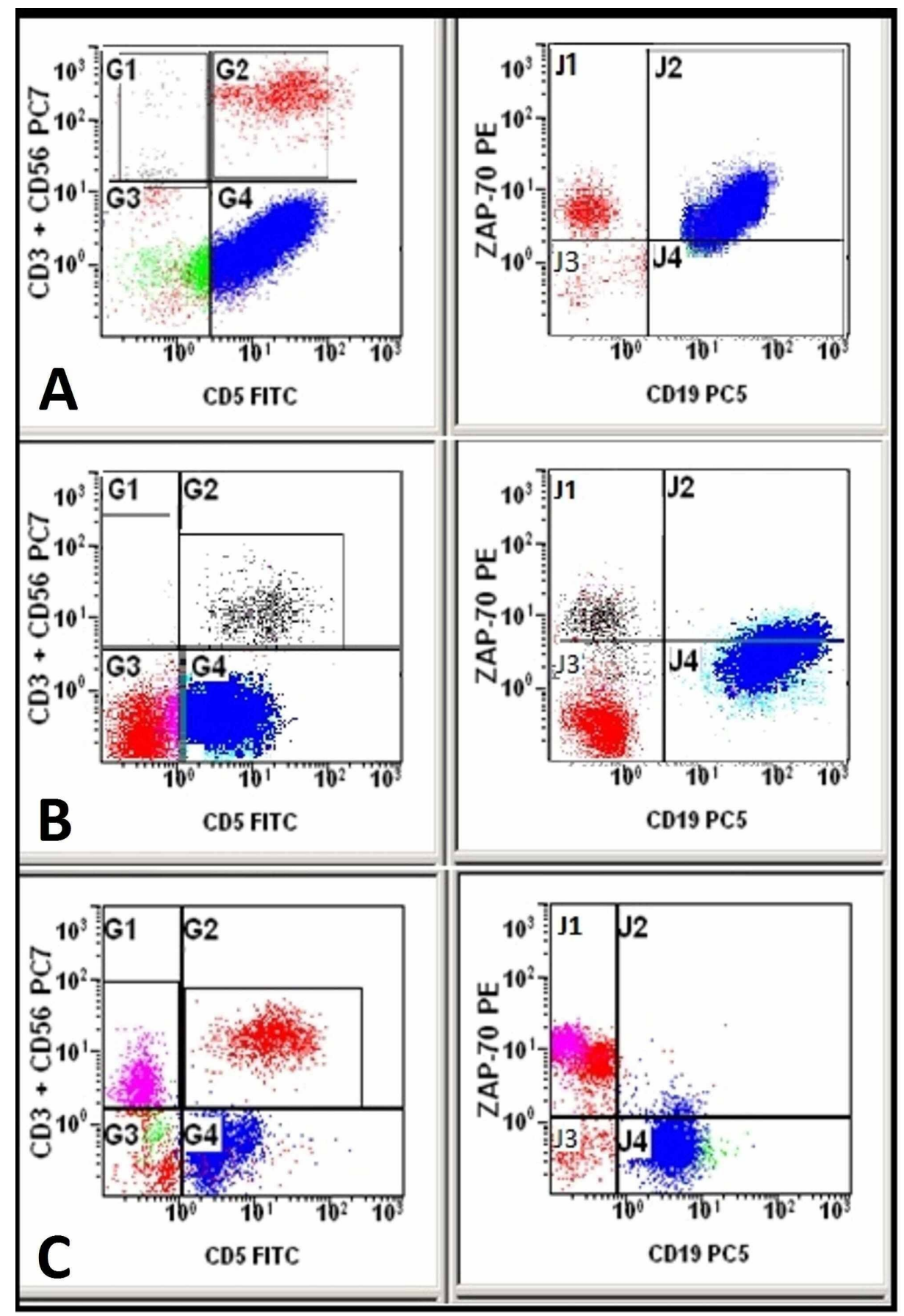

FIGURE 1: Dot plots showing normal B-cells (CD19+, CD5-) in green, Tcells (CD19-, CD5+, CD3+) in red, and CLL cells (CD19+, CD5+) in blue. ZAP-70 expression is high (A), low (B), and negative (C) in CLL cells (J2 quadrant)

\section{Discussion}

Chronic lymphocytic leukemia is a heterogeneous disease with widely variable outcomes. About one-third of the patients have an indolent disease with prolonged survival up to 20 years. The eventual cause of death in these patients is usually unrelated to CLL [11]. Some patients initially have a benign course extending over a period of five to 10 years followed by an aggressive phase. In its most aggressive form either following a benign course or upfront from the time of diagnosis, patients die within three to five years of diagnosis due to complications directly relatable to CLL $[12,13]$.

The Rai and Binet staging systems take into account the degree of anemia, thrombocytopenia, lymphocytosis, lymph node involvement, and visceromegaly [14]. Both these systems are helpful for the 
clinical care of the patients and are in wide use for the past several years. However, considering the highly variable natural history of the disease, several laboratory parameters have also been described which include ZAP-70 and CD38 expression, beta- 2 microglobulin levels, mutational status of IgHv, and other genetic abnormalities [4]. In most studies, ZAP-70 expression is reported as positive and negative, however, a large cohort lies in a gray zone with values as low as to be close to negative or as high as encroaching positive territory so called gray zone. This study provides information on ZAP-70 expression into three well-defined categories, high (positive), low (intermediate), and negative. The information is useful for the physician for treatment decisions and counseling patients right at the time of diagnosis.

B-CLL is twice as common in males than females; this fact was reflected exactly in our study as well with male to female ratio of $2: 1$ [15]. However, the median age at diagnosis in the current study was 62 years as compared to 72 years reported in western literature $[11,16]$. No statistically significant difference in Z-index was found with respect to the gender or age of the patients (Tables 1,2).

A wide variation in ZAP-70 expression in B-CLL has been observed in studies done in various regions of the world. A previous Pakistani study reported a prevalence of $13.5 \%$ [17]. A prevalence of $25 \%$ was reported from India with very similar genetics and life-style similarities [9]. Both these studies used a cutoff of $20 \%$ for defining positive ZAP-70 expression. In some earlier analyses, ZAP-70 prevalence as high as 57\% with a cut-off of $20 \%$ has also been described $[18,19]$. In the current analysis, a high Z-index was observed in $15 \%$ of patients with a cut-off of $27 \%$. However, a significant proportion (41.6\%) fell in the gray zone.

Although, the variation in the prevalence of ZAP-70 expression in different populations can be attributed to differences in genetics, ethnic origin, geographical and environmental factors, a large proportion of reports have used arbitrary threshold values to define ZAP-70 positivity [9,20]. In our study, we used Z-index to determine ZAP-70 expression rather than using simple quadrant analyses set by using isotype controls. Zindex incorporates ZAP-70 MFI signals in normal B-cells (negative control) in addition to T-cell MFI (positive control). This method is more sensitive and reproducible. Earlier studies have described ZAP-70 expression either as positive or negative using arbitrary cut-offs; mostly 20\% [9]. This approach is likely to miss some cases with decreased ZAP-70 expression and hence important prognostic information. We adopted a Z-index strategy to quantify ZAP-70 from a landmark study for its merits [10]. In addition to standardized instrumentation setup and data analysis, unique sample fixation and permeabilization techniques have also been described in this study. The authors claim an 18- to 22-fold difference in the level of ZAP-70 protein measured in normal T-cells (ZAP-70 positive) as compared to normal B-cells (ZAP-70 negative). An evaluation of intra- and interlaboratory performance of this optimized assay in the analysis of CLL patient material has also been described in the analysis.

The advantages of ZAP-70 analysis as a predictor of prognosis in B-CLL include stability in its level over time. In one study with a median interval time of 25 months (4-179) in between sequential sampling, no changes in the pattern of ZAP-7- staining were observed in any case [20]. The flow cytometric analysis is a lot simpler, faster, and cheaper than PCR, sequencing, or western blotting which are used for IGHV mutation. As ZAP-70 is also expressed by T-lymphocytes and natural killer cells, careful separation of CLL B-cells is important. This can readily be achieved by appropriate labeling of lymphocyte-specific markers and appropriate gating strategies. Furthermore, due to its strong correlation, ZAP-70 expression analysis is an important surrogate marker for establishing IGHV mutational status particularly in developing countries where more sophisticated molecular techniques are unavailable.

The main limitation of our study is the lack of clinical follow-up primarily for the reason that a large number of specimens are outside referrals. However, the strength of the study is the use of Z-index rather than mere use of ZAP-70 percentage. Most other studies have utilized ZAP-70 percentages with arbitrary cut-offs.

Using the Z-index strategy, our study highlights an important prognostic sub-category of "low or borderline or gray zone or indeterminate ZAP-70" expression in addition to distinct "high" and "negative" categories and it is precisely this significantly large group which need further research and insight.

\section{Conclusions}

This study showed only 15\% of B-CLL cases showing high ZAP-70 expression, a surrogate biomarker for possible aggressive behavior which may necessitate therapeutic intervention and close surveillance.

\section{Additional Information \\ Disclosures}

Human subjects: Consent was obtained by all participants in this study. Ethical Review Committee, Aga Khan University Hospital issued approval 0404-290. Approval of the research study was accepted as exempt because the collection or study of existing data, documents, records, pathological specimens, or diagnostic specimens were recorded by the investigator in such a manner that subjects cannot be identified, directly or through identifiers linked to the subjects. Animal subjects: All authors have confirmed that this study did not involve animal subjects or tissue. Conflicts of interest: In compliance with the ICMJE uniform disclosure form, all authors declare the following: Payment/services info: All authors have declared that no 
financial support was received from any organization for the submitted work. Financial relationships: All authors have declared that they have no financial relationships at present or within the previous three years with any organizations that might have an interest in the submitted work. Other relationships: All authors have declared that there are no other relationships or activities that could appear to have influenced the submitted work.

\section{References}

1. Siegel RL, Miller KD, Jemal A: Cancer statistics, 2020. CA Cancer J Clin. 2020, 70:7-30. 10.3322/caac.21590

2. Kwok M, Rawstron AC, Varghese A, et al.: Minimal residual disease is an independent predictor for 10-year survival in CLL. Blood. 2016, 128:2770-2773. 10.1182/blood-2016-05-714162

3. Pulte D, Castro FA, Jansen L, et al.: Trends in survival of chronic lymphocytic leukemia patients in Germany and the USA in the first decade of the twenty-first century. J Hematol Oncol. 2016, 9:28. 10.1186/s13045016-0257-2

4. Brown JR, Hillmen P, O'Brien S, et al.: Extended follow-up and impact of high-risk prognostic factors from the phase 3 RESONATE study in patients with previously treated CLL/SLL. Leukemia. 2018, 32:83-91. 10.1038/leu.2017.175

5. Taghiloo S, Allahmoradi E, Ebadi R, et al.: Upregulation of galectin-9 and PD-I1 immune checkpoints molecules in patients with chronic lymphocytic leukemia. Asian Pac J Cancer Prev. 2017, 18:2269-2274. 10.22034/APTCP.2017.18.8.2269

6. Basabaeen AAM, Abdelgader EA, Babekir EA, et al.: TP53 gene 72 arg/pro (rs1042522) single nucleotide polymorphism contribute to increase the risk of b-chronic lymphocytic leukemia in the Sudanese population. Asian Pac J Cancer Prev. 2019, 20:1579-1585. 10.31557/APJCP.2019.20.5.1579

7. Wiestner A, Rosenwald A, Barry TS, et al.: ZAP-70 expression identifies a chronic lymphocytic leukemia subtype with unmutated immunoglobulin genes, inferior clinical outcome, and distinct gene expression profile. Blood. 2003, 101:4944-4951. 10.1182/blood-2002-10-3306

8. Au-Yeung BB, Shah NH, Shen L, Weiss A: ZAP-70 in signaling biology, and disease. Annu Rev Immunol. 2018, 36:127-156. 10.1146/annurev-immunol-042617-053335

9. Gogia A, Sharma A, Raina V, Kumar L, Gupta R, Kumar R: Prevalence of ZAP-70 and CD 38 in Indian chronic lymphocytic leukemia patients. Indian J Cancer. 2013, 50:333-336. 10.4103/0019-509X.123620

10. Shankey TV, Forman M, Scibelli P, et al.: An optimized whole blood method for flow cytometric measurement of ZAP-70 protein expression in chronic lymphocytic leukemia. Cytometry B Clin Cytom. 2006, 70:259-269. 10.1002/cyto.b.20135

11. Strati P, Parikh SA, Chaffee KG, et al.: Relationship between co-morbidities at diagnosis, survival and ultimate cause of death in patients with chronic lymphocytic leukaemia (CLL): a prospective cohort study. Br J Haematol. 2017, 178:394-402. 10.1111/bjh.14785

12. Greipp PT, Smoley SA, Viswanatha DS, et al.: Patients with chronic lymphocytic leukaemia and clonal deletion of both 17p13. 1 and 11q22. 3 have a very poor prognosis. Br J Haematol. 2013, 163:326-333. 10.1111/bjh.12534

13. Rossi D, Rasi S, Spina V, et al.: Integrated mutational and cytogenetic analysis identifies new prognostic subgroups in chronic lymphocytic leukemia. Blood. 2013, 121:1403-1412. 10.1182/blood-2012-09-458265

14. Hallek M: Chronic lymphocytic leukemia: 2020 update on diagnosis, risk stratification and treatment . Am J Hematol. 2019, 94:1266-1287. 10.1002/ajh.25595

15. Beiggi S, Banerji V, Deneka A, Griffith J, Gibson SB, Johnston JB: Comparison of outcome of patients with CLL who are referred or nonreferred to a specialized CLL clinic: a Canadian population-based study. Cancer Med. 2016, 5:971-979. 10.1002/cam4.559

16. Al-Sawaf O, Robrecht S, Bahlo J, et al.: Richter transformation in chronic lymphocytic leukemia (CLL)-a pooled analysis of German CLL Study Group (GCLLSG) front line treatment trials. Leukemia. 2020, 1-8. 10.1038/s41375-020-0797-X

17. Zeeshan R, Irfan SM, Sultan S, Bhimani S: ZAP-70 protein expression in B-cell chronic lymphoid leukemia: a single center experience from Pakistan. Asian Pac J Cancer Prev. 2015, 16:1587-1590. 10.7314/apjcp.2015.16.4.1587

18. Schroers R, Griesinger F, Trümper L, et al.: Combined analysis of ZAP-70 and CD38 expression as a predictor of disease progression in B-cell chronic lymphocytic leukemia. Leukemia. 2005, 19:750-758. 10.1038/sj.leu.2403707

19. Crespo M, Bosch F, Villamor N, et al.: ZAP-70 expression as a surrogate for immunoglobulin-variable-region mutations in chronic lymphocytic leukemia. N Engl J Med. 2003, 348:1764-1775. 10.1056/NEJMoa023143

20. Carreras J, Villamor N, Colomo L, et al.: Immunohistochemical analysis of ZAP-70 expression in B-cell lymphoid neoplasms. J Pathol. 2005, 205:507-513. 10.1002/path.1727 